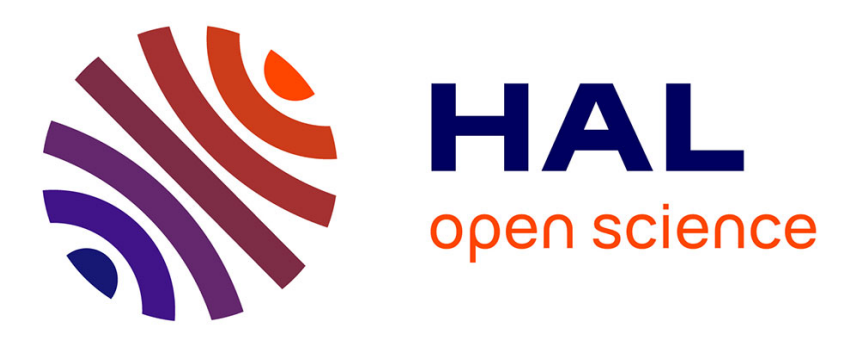

\title{
Versatile digital micromirror device-based method for the recording of multilevel optical diffractive elements in photosensitive chalcogenide layers (AMTIR-1)
}

Alexandre Joerg, Mael Vigneaux, Julien Lumeau

\section{- To cite this version:}

Alexandre Joerg, Mael Vigneaux, Julien Lumeau. Versatile digital micromirror device-based method for the recording of multilevel optical diffractive elements in photosensitive chalcogenide layers (AMTIR-1). Optics Letters, 2016, 41, pp.3415-3418. 10.1364/OL.99.099999 hal-01359464

\author{
HAL Id: hal-01359464 \\ https://hal.science/hal-01359464
}

Submitted on 13 Mar 2019

HAL is a multi-disciplinary open access archive for the deposit and dissemination of scientific research documents, whether they are published or not. The documents may come from teaching and research institutions in France or abroad, or from public or private research centers.
L'archive ouverte pluridisciplinaire HAL, est destinée au dépôt et à la diffusion de documents scientifiques de niveau recherche, publiés ou non, émanant des établissements d'enseignement et de recherche français ou étrangers, des laboratoires publics ou privés. 


\title{
Versatile Digital Micromirror Device-based method for the recording of multilevel optical diffractive elements in photosensitive chalcogenide layers (AMTIR-1)
}

\author{
Alexandre Joerg, ${ }^{1}$ Mael Vignaux, ${ }^{1}$ And Julien lumeau ${ }^{1, *}$ \\ ${ }^{1}$ Aix Marseille Université, CNRS, Centrale Marseille, Institut Fresnel UMR 7249, 13013 Marseille, France \\ *Corresponding author: julien.lumeau@fresnel.fr
}

Received XX Month XXXX; revised XX Month, XXXX; accepted XX Month XXXX; posted XX Month XXXX (Doc. ID XXXXX); published XX Month XXXX

\begin{abstract}
A new alternative and versatile method for the production of Diffractive Optical Elements (DOEs) with up to 4 phase levels in AMTIR-1 ( $\left.\mathrm{Ge}_{33} \mathrm{As}_{12} \mathrm{Se}_{55}\right)$ layers is demonstrated. The developed method proposes the use of the photosensitive properties of the layers and a specific in-situ optical monitoring coupled with a reverse engineering algorithm to control the trigger points of the writing of the different diffractive patterns. Examples of various volume DOEs are presented. (C) 2016 Optical Society of America
\end{abstract}

OCIS codes: (160.5335) Photosensitive materials; (050.1970); Diffractive optics; (310.6845) Thin film devices and applications.

http://dx.doi.org/10.1364/OL.99.099999

Chalcogenide glasses (ChG) are composed from a chalcogen element such as Sulphur, Selenium, and Tellurium. To create a glass network, some other atoms are added such as As, Ge, Sb, Ga, Si or P. ChG are particularly interesting due to their specific optical properties. In fact they are transparent into the mid-infrared up to $20 \mu \mathrm{m}$ depending on their chemical composition [1]. The refractive index is high $\mathrm{n} \sim 2-3$ and ChG possess also a high nonlinear refractive index $\mathrm{n}_{2}$ [2]. But one of the most striking property is their photosensitivity. Using light exposure with a wavelength below the optical bandgap, the chemical bonds can be rearranged. This effect directly modifies the optical properties of the ChG. This very specific property has led to different kinds of applications such as phase change memory [3]... As thin films, ChG were thoroughly studied and the impact of inserting a photosensitive layer into an optical filter to modify its spectral response was demonstrated [4]. As an example, it was shown, a few years ago, that it is possible to use the photo-bleaching or photo-darkening [5] effects to adjust the central wavelength of a Fabry-Perot filter and therefore generate ultra-uniform bandpass filters [6].

The ChG studied in this paper is AMTIR-1. It is composed in molar percent with $33 \%$ of germanium, $12 \%$ of arsenic and $55 \%$ of selenium and was provided by Amorphous Material Company. AMTIR-1 was already deposited as thin films by pulsed laser deposition [7], thermal or e-beam evaporation, and showed excellent optical properties [8]. Layers from AMTIR-1 were shown to be excellent candidates for the production of various optical elements such as ring resonators [9]... We recently showed that these layers are also a good candidate for the production of a new kind of optical elements [10]. Actually with the theoretical and experimental developments of diffractive optical elements, metamaterials or metasurfaces, there is a higher and higher demand for 3D micro- and nanostructuring of multilayer stacks. 1Dstructures are easily obtained by the optical coating technology, while 3D-structures require micro- or nano-engineering the local thickness of the deposited structures using lithographic processes combined with etching techniques. We showed that for coarse structures, i.e. for structures having a pitch with size much larger than the wavelength (i.e. with size larger than $10 \mu \mathrm{m}$ ), it is possible to create volume phase structures by locally controlling the local refractive index of a photosensitive layer. Such an approach was applied to the fabrication of binary DOEs. However, it is well known that for many applications and in order to achieve higher diffraction efficiency, especially for the production of asymmetrical beams, the use of multi-level DOEs is mandatory. In this paper we demonstrate the possibility to photostructure a photosensitive chalcogenide layer with 4 levels in order to produce a Volume Diffractive Optical Element (VDOE). Methods of production and monitoring are detailed.

The chalcogenide layers and thin film stacks were produced using electron beam physical vapor deposition in a Balzers BAK600 chamber. The residual pressure inside the vacuum chamber was around $\sim 10^{-6}$ mbar and the deposition rate was $10 \pm 1 \mathrm{~A} / \mathrm{s}$. In a previous works [8] using those parameters, we first demonstrated that AMTIR-1 materials provide broad transparency range above $850 \mathrm{~nm}$, high refractive index $(n=2.74 @ 1 \mu \mathrm{m})$ and photosensitive properties such as photo-bleaching effect. This effect occurs into the AMTIR-1 layers when exposed to a light with a wavelength whose energy is below the bandgap. This effect allows generating refractive index decrement down to -0.04 at $1 \mu \mathrm{m}$ that was then applied to binary DOEs. Moreover, it was shown that this refractive index change is 
permanent and is stable with time over extended period of time of 10 weeks (the duration of the test). Therefore AMTIR-1 based layers are compatible with the production of stable optical elements.

Using those previous results, the goal of this work was to show the possibility to further structure these photosensitive layers and then to generate a multilevel DOEs with up to 4 levels for a wavelength around $850 \mathrm{~nm}$ i.e. the nearest wavelength from the optical bandgap of the material $(\mathrm{Eg}=1.42 \mathrm{eV})$ that can still secure high transmission. This wavelength was chosen in order to make the best use of the photobleaching effect, i.e. maximize the photo-induced refractive index change and minimize the required refractive index change to obtain a $2 \pi$ phase shift. A 4 levels VDOE required to get $3 \pi / 2$ as maximum phase level which means, with a refractive index variation equal to $\Delta \mathrm{n}$, a thickness tof:

$$
\mathrm{t}=\frac{3}{4} \frac{\lambda}{\Delta \mathrm{n}}
$$

We opted for an AMTIR-1 layer with thickness of $\sim 23 \mu \mathrm{m}$ deposited between two AR-coating stacks which annihilate losses due to Fresnel coefficients and oscillations caused by the interferometric nature of a single layer. AR-coatings were made of two layers of tantalum pentoxide ( $\mathrm{n} \sim 2.1$ ) and silicon dioxide ( $\mathrm{n} \sim 1.46)$. The designed multilayer structure is therefore:

$$
\text { Substrate/AR2/AMTIR-1/AR1/ Air }
$$

To record VDOEs in a photosensitive layer, an optical setup was designed to perform the exposure. A detailed description can be found in [10].

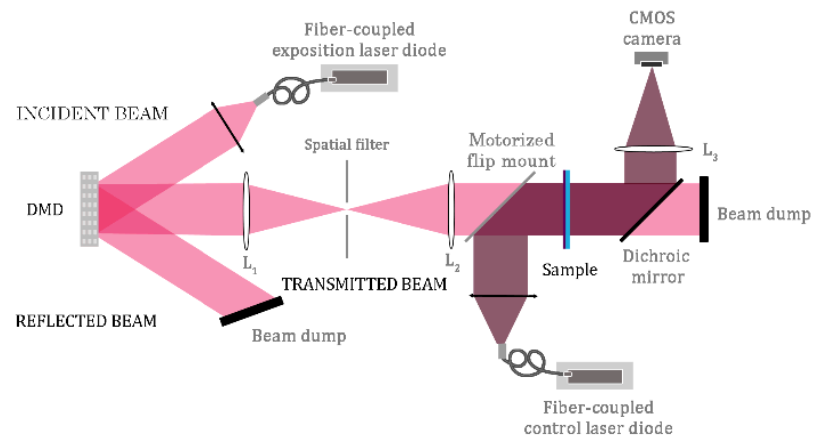

Fig. 1: Experimental setup for patterning light and monitoring DOE writing.

In short, this set-up (Fig. 1) is based on a Digital Micromirror Device (DMD) which is shined by a collimated laser diode beam with central wavelength at $808 \mathrm{~nm}$, a spectral width of $4 \mathrm{~nm}$ and a power density of $15 \mathrm{~W} / \mathrm{cm}^{2}$. According to the extinction coefficient of AMTIR-1 layers at $808 \mathrm{~nm}$, this wavelength was chosen to secure large enough absorption to trigger the AMTIR-1 photosensitivity and keep almost uniform exposure throughout the chalcogenide-layer thickness ( $\mathrm{T}_{\text {ouput }}$ $=50 \%$ after propagation within a $23 \mu \mathrm{m}$ thick layer). The DMD was used to pattern the exposure intensity following a given profile loaded in the software, and was then imaged on the sample plane with a 4-f imaging system (Fig. 1: pink color). Intensity spatial profiles can then be adapted to the application, i.e., in our case, the generation of diffractive patterns.

A second optical (Fig. 1: purple color) system acts as an in-situ optical monitoring that is analogous to what is classically used for the monitoring of thin film filters. It is mainly composed by a fiber coupled laser diode centered at $850 \mathrm{~nm}$ (collimated with waist beam $\omega \sim 1.3 \mathrm{~mm}$ ), a $500 \mathrm{~mm}$ focal lens and a CMOS camera. The monitoring wavelength is identical to the one that was used to design the VDOEs. During the exposure, this optical system measures at different times $t$ the evolution of the intensity distribution in the focal plan of a lens i.e. in the far field. Thus it allows to monitor the DOE diffracted intensity during its fabrication of and to define trigger points when exposure must be stopped, i.e. when a given phase level has been reached. Such an approach was already implemented for the fabrication of binary VDOEs [9]; exposure is switched off when the far field measured intensity is similar to the modeled one, meaning that the $\pi$ phase is met.

For a 4-graylevel DOE which includes $0, \pi / 2, \pi ; 3 \pi / 2$ as phase levels, the exposure and monitoring strategy is quite the same. But the exposure arrangement was chosen in order to have characteristic farfield intensity distributions after exposing each gray-level. In fact the impact, on the far-field distribution intensity, of a DOE with a single $\pi / 2$ phase level is not enough to produce a significant change that can be monitored with the in-situ optical monitoring. This results can be confirmed by calculating the theoretical field distribution in the focal plan of lens expressed by:

$$
U_{f}(\mathrm{u}, \mathrm{v})=\frac{\exp \left(\mathrm{j} \frac{\mathrm{k}}{2 \mathrm{f}}\left(\mathrm{u}^{2}+\mathrm{v}^{2}\right)\right)}{\mathrm{j} \lambda \mathrm{f}} \mathrm{TF}\left[\mathrm{u}_{\text {gauss }} \cdot \exp \left(\mathrm{j} \varphi_{\mathrm{DOE}}(x, y)\right)\right]
$$

where ugauss is the Gaussian field TM00 from the optical monitoring and $\varphi_{\text {DOE }}$ the DOE phase pattern with 4 levels phase. To overcome this effect, a dedicated strategy was developed.

- $\quad$ First the phase levels over $\pi$ are exposed until reaching the desired phase change. We obtain the equivalent of a binary DOE.

- $\quad$ Then the area that not yet been exposed and corresponding to $\pi / 2$ levels are exposed until reaching the desired phase change

- $\quad$ Finally the area that have been exposed to with $\pi$ phase shift and that are associated with $3 \pi / 2$ levels are further exposed in order to increment the level by an additional $\pi / 2$ level until getting the desired final intensity distribution.

Thus the phase pattern exposed is divided into 3 parts, as the following expression:

where

$$
\varphi_{D O E}(x, y)=\pi \varphi_{1}+\frac{\pi}{2} \varphi_{2}+\frac{\pi}{2} \varphi_{3}
$$

$$
\left\{\begin{array}{l}
\varphi_{1}(x, y)= \begin{cases}1 & \text { if } \varphi_{\mathrm{DOE}}(x, y) \geq \pi \\
0 & \text { otherwise }\end{cases} \\
\varphi_{2}(x, y)= \begin{cases}1 & \text { if } \varphi_{\mathrm{DOE}}(x, y)=\frac{\pi}{2} \\
0 & \text { otherwise }\end{cases} \\
\varphi_{3}(x, y)= \begin{cases}1 & \text { if } \varphi_{\mathrm{DOE}}(x, y)=\frac{3 \pi}{2} \\
0 & \text { otherwise }\end{cases}
\end{array}\right.
$$

An example of the three different phase profiles in case of the fabrication of a 4-level 4 beams splitter is illustrated in Fig. 2.
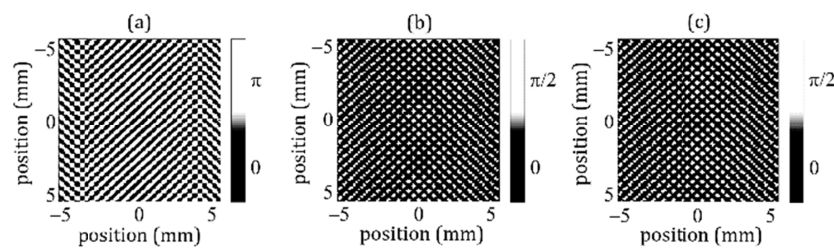

Fig. 2: Phase masks for a step by step exposure: (a) phase level over $\pi$, (b) phase level equal to $\pi / 2$, (c) phase level equal to $3 \pi / 2$. 
This procedure enables to have characteristic intensity distributions for each phase level in the focal plane of the lens during optical monitoring and thus to precisely control each step. An example of the modeled characteristic far field intensity when recording a $2 \times 2$ beam splitter is illustrated in Fig. 4. (a)

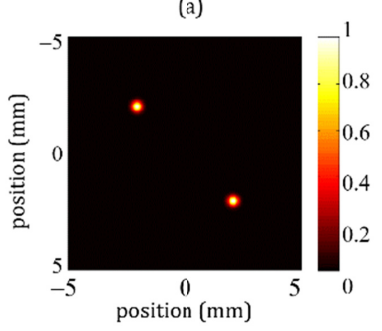

(b)

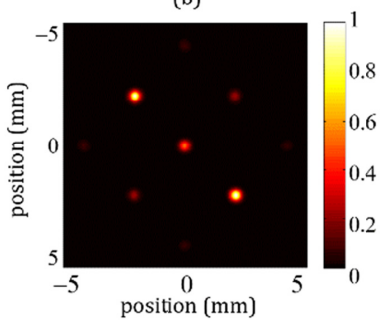

(c)

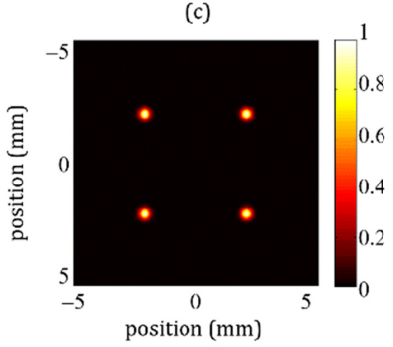

Fig. 3: Simulation of far-field diffraction patterns of a Gaussian beam after propagation through different graylevel phase mask at the focal plane of a $500 \mathrm{~mm}$ lens: (a) phase level over $\pi$, (b) phase level equal to $\pi / 2$ plus phase level over $\pi$, (c) the final pattern.

Very specific and characteristic intensity profiles are associated with the creation of each level: the first step ( $\pi$ level) splits the beam into two new beams, the second intermediate state ( $\pi / 2$ level) is associated with the appearance of 2 new additional beams as well as the reappearance of the zero order beam, and finally the last level $(3 \pi / 2$ level) permits to generate the four new beams with equal intensity and low zero order beam.

Similarly, Figure 4 shows an example (modeling) of the proposed exposure and monitoring strategy after exposing the first two levels and the third level with a $\pi / 4$ phase value of square top-hat beam. Its associated vizualization shows the overall strategy throughout the exposure of all levels.
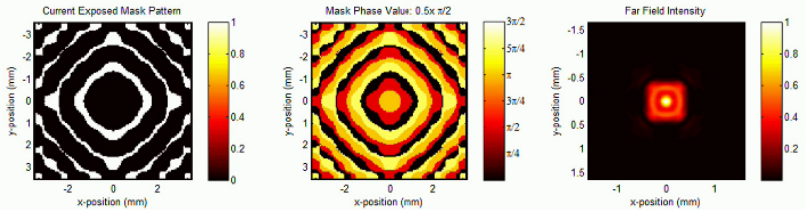

Fig. 4: Illustration of the exposure and monitoring strategy after exposing the first two levels and the third level with a $\pi / 4$ phase value square top-hat beam (Figure) and troughout the fabrication process (visualization).

One may wonder why a 3-step exposure is necessary, while the usual processes, such as photolithographic process, require only two exposure steps. One for $\pi$ phase level and another to add $3 \pi / 2$ and $\pi / 2$ levels. Actually, with a photosensitive chalcogenide layer, due to the non-linear response of the photo-bleaching effect, we found that it is advantageous to have a 3-step exposure as the kinetics of refractive index change will be different for the second and fourth level [10].
Finally to precisely control the evolution of the diffractive pattern during the exposure, a reverse engineering method was added. It compares the measured far-field intensity profile in the focal plane of a $500 \mathrm{~mm}$ lens, at time $t$, and a simulated profile which phase change is optimized in order to minimize merit function defined by:

$$
E F=\sqrt{\frac{1}{M N} \sum_{u, v}^{M, N}\left(I_{m e s}(u, v)-I_{t h}(u, v)\right)^{2}}
$$

where

$$
\begin{aligned}
I_{t h}(u, v)= & \frac{1}{\lambda^{2} f^{2}} \mid T F\left[u _ { \text { gauss. } } \cdot \operatorname { e x p } \left(j \alpha \pi \varphi_{1}+j \beta \frac{\pi}{2} \varphi_{2}+\right.\right. \\
& \left.\left.j \gamma \frac{\pi}{2} \varphi_{3}\right)\right]\left.(u, v)\right|^{2}
\end{aligned}
$$

The phase components $\varphi_{1}, \varphi_{2}$ and $\varphi_{3}$ are those defined in equation (4), the coefficient $\alpha, \beta$ and $\gamma$ are between 0 and 1 and optimized by the algorithm that was developed using Matlab. As the exposure is made step by step each coefficient is estimated one by one and the value are fixed at the end of each exposure step. Using this extracted value of the phase during exposure, it is also possible to estimate the necessary remaining exposure during of each step.

Different 4-Level VDOEs have been fabricated following the experiment protocol previously described. In this section we present the results of a $2 \times 2$ beam splitter and a square top hat beam splitter. The VDOEs patterns have been designed either by a usual iterative Fourier transform algorithm [12,13] or a simulated annealing algorithm [14]. Then, they were recorded using the multi-step procedure. The result at achievement obtained for the $2 \times 2$ beam splitter is illustrated in Fig. 5 and the results for the top-hat are illustrated in Fig. 6, Fig. 7 and Fig. 8, the figures corresponding respectively to the diffraction after exposing the $\pi$-levels, $\pi$ and $\pi / 2$ levels and $\pi, \pi / 2$ and $3 \pi / 2$ levels. As one see, there is a good correlation between theoretical results and experimental one. Moreover coefficients $\alpha, \beta$, and $\gamma$ calculated using the Matlab algorithm are over 0.95 confirming the good creation of the VDOE pattern in both examples.
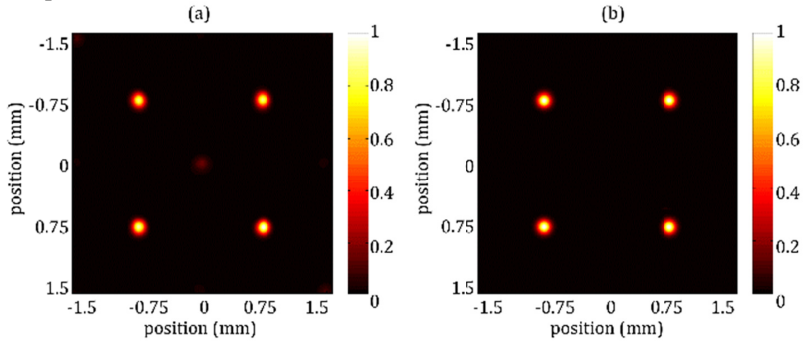

Fig. 5: Far-field diffraction patterns of a Gaussian beam after propagation through a graylevel phase mask at the focal plane of a $500 \mathrm{~mm}$ lens: (a) experimental results, (b) theoretical results.
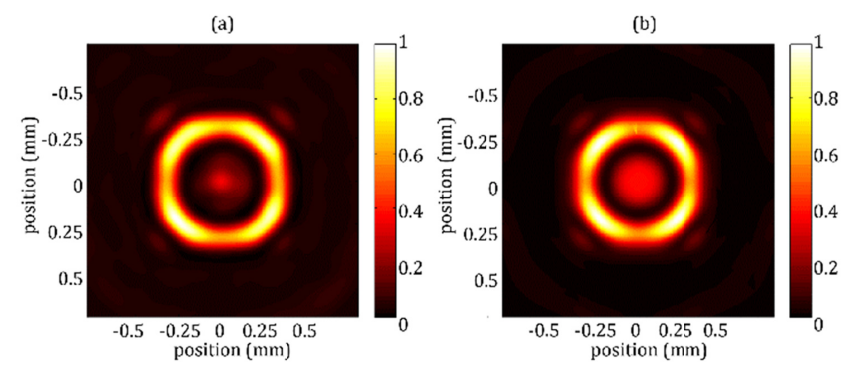
Fig. 6: Far-field diffraction patterns of a Gaussian beam after propagation through the first exposed mask (graylevel $\geq \pi$ ) at the focal plane of a $500 \mathrm{~mm}$ lens: (a) experimental results, (b) theoretical results.
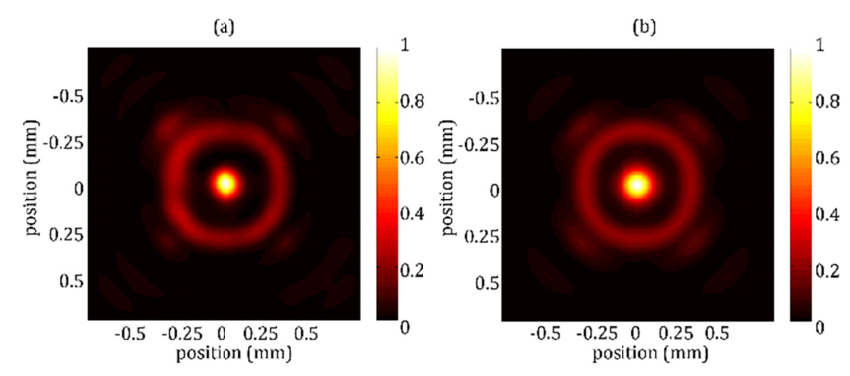

Fig. 7: Far-field diffraction patterns of a Gaussian beam after propagation through the second exposed (graylevel $\geq \pi+$ graylevel $=$ $\pi / 2$ ) mask at the focal plane of a $500 \mathrm{~mm}$ lens: (a) experimental results, (b) theoretical results.

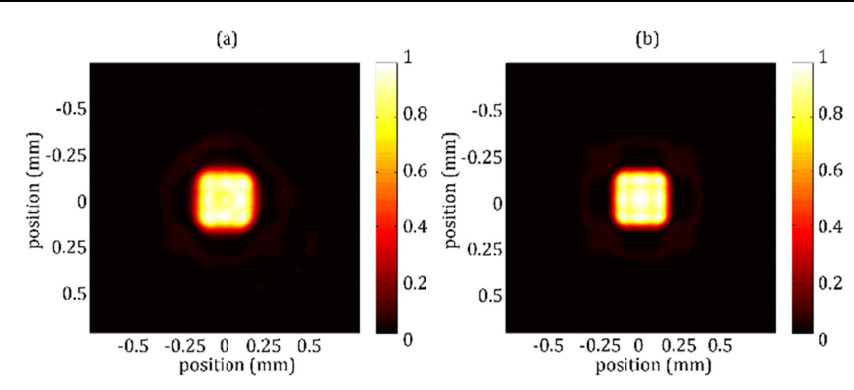

Fig. 8: Far-field diffraction patterns of a Gaussian beam after propagation through the final 4 graylevels phase mask at the focal plane of a $500 \mathrm{~mm}$ lens: (a) experimental results, (b) theoretical results.

To characterize the results, the diffraction efficiency was estimated for both VDOEs using the formula:

$$
\text { eff }=\frac{I_{D O E}}{I_{\text {Gauss }}}
$$

Where $I_{D O E}$ is the intensity contained in the region of interest i.e. for the $2 \times 2$ beam splitter the intensity integrated over the 4 points and for the top-hat the intensity integrated over the square beam profile. $\mathrm{I}_{\text {Gauss }}$ is the intensity integrated over the whole initial Gaussian beam aperture after propagation through a uniform part of the AMTIR-1 coated glass plate in the focal place of the same lens. This intensity thus takes into account the loss induced by Fresnel reflection and the absorption of the AMTIR-1 layer. Such a definition allows characterizing the efficiency of the fabricated patterns and not intrinsic losses of the optical element. Results are summarized in Table 1. One can see that efficiency is still limited because the designs were optimized in order to show very characteristic diffraction patterns when exposing each gray-level and therefore validate the concept detailed in this paper. This efficiency could then be highly improved by optimizing the designs.

Finally an additional characterization of the $2 \times 2$ beam splitter was performed by calculating the residual intensity inside the zero-order. It use the same formula (5) but IDOE is replaced by the Izero corresponding to intensity of the zero-order. The results give a residual intensity equal to $1 \%$.
Table 1. Diffraction Efficiency and zero order intensity of the fabricated DOEs.

\begin{tabular}{|c|c|c|}
\hline & Efficiency & Zero-Order \\
\hline $\mathbf{2} \times \mathbf{2}$ beam splitter & $70 \%$ & $1 \%$ \\
\hline Top-Hat & $80 \%$ & N/A \\
\hline
\end{tabular}

We have presented here a comprehensive description of the fabrication of a multilevel VDOE using closed-loop in situ optical monitoring. Two examples were detailed. The first one is a $2 \times 2$ beam splitter and the second one is a square top-hat beam converter with 4 -levels. These results validate a versatile and efficient way for the recording of volume multi-level diffractive optical elements.

\section{References}

1. A. Zakery and S. Elliott, Journal of Non-Crystalline Solids 330, 1-12 (2003).

2. T.I. Kosa, R. Rangel-Rojo, E. Hajto, P.J.S. Ewen, A.E. Owen, A.K. Kar, and B.S. Wherrett, Journal of Non-Crystalline Solids 166, 1219-1222 (1993).

3. H.-S. Wong, S. Raoux, S. Kim, J. Liang, J. P. Reifenberg, B. Rajendran, M. Asheghi and K. E. Goodson, Proceedings of the IEEE 98, 2201-2227 (2010).

4. M. Lequime and J. Lumeau, Proc. SPIE of the Advances in Optical ThinFilms conference (Jena, Germany), paper 5963-08, September 2005.

5. A. v. Popta, R. DeCorby, C. Haugen, T. Robinson and J. McMullin, Optics Express 10, 639-644 (2002).

6. W. Shen, M. Cathelinaud, M. Lequime, V. Nazabal and X. Liu, Optics communications 281, 3726-3731 (2008).

7. R. A. Jarvis, R. P. Wang, A. V. Rode, C. Zha and B. Luther-Davies, Journal of Non-Crystalline Solids 353, 947-949 (2007).

8. A. Joërg, F. Lemarchand, M. Zhang, M. Lequime, and J. Lumeau, Journal of Non-Crystalline Solids 442, 22-28 (2016).

9. P. Ma, D.-Y. Choi, Y. Yu, Z. Yang, K. Vu, T. Nguyen, A. Mitchell, B. Luther-Davies, and S. Madden, Opt. Express 23, 19969-19979 (2015).

10. A. Joërg and J. Lumeau, Opt. Lett. 40, 3233-3236 (2015).

11. J. Lumeau and L. B. Glebov, Applied Optics 52(17), 3993-3997 (2013).

12. C. Kress and P. Meyrueis, Applied Digital Optics: From Micro-optics to Nanophotonics, Wiley, 2009.

13. O. Ripoll, V.Kettunen and H. Herzig, Optical Engineering 43, 2549-2556 (2004).

14. N. Yoshikawa and T. Yatagai, Applied optics 33, 863-868 (1994). 


\section{References}

1. A. Zakery and S. Elliott, "Optical properties and applications of chalcogenide glasses: a review," Journal of Non-Crystalline Solids 330, 1-12 (2003).

2. T.I. Kosa, R. Rangel-Rojo, E. Hajto, P.J.S. Ewen, A.E. Owen, A.K. Kar, and B.S. Wherrett, "Nonlinear optical properties of silver-doped As2S3", Journal of Non-Crystalline Solids 166, 1219-1222 (1993).

3. H.-S. Wong, S. Raoux, S. Kim, J. Liang, J. P. Reifenberg, B. Rajendran, M. Asheghi and K. E. Goodson, "Phase Change Memory," Proceedings of the IEEE 98, 2201-2227 (2010).

4. M. Lequime and J. Lumeau, "Laser trimming of thin-film filters", Proc. SPIE of the Advances in Optical Thin-Films conference (Jena, Germany), paper 5963-08, September 2005.

5. A. v. Popta, R. DeCorby, C. Haugen, T. Robinson and J. McMullin, "Photoinduced refractive index change in As2Se3 by $633 \mathrm{~nm}$ illumination", Optics Express 10, 639-644 (2002).

6. W. Shen, M. Cathelinaud, M. Lequime, V. Nazabal and X. Liu, "Photosensitive post tuning of chalcogenide Te20As30Se50 narrow bandpass filters", Optics communications 281, 3726-3731 (2008).

7. R. A. Jarvis, R. P. Wang, A. V. Rode, C. Zha and B. Luther-Davies, "Thin film deposition of Ge33As12Se55 by pulsed laser deposition and thermal evaporation: Comparison of properties", Journal of Non-Crystalline Solids 353, 947-949 (2007).

8. A. Joërg, F. Lemarchand, M. Zhang, M. Lequime, and J. Lumeau, "Optical characterization of photosensitive AMTIR-1 chalcogenide thin layers deposited by electron beam deposition", Journal of Non-Crystalline Solids 442, 22-28 (2016).

9. P. Ma, D.-Y. Choi, Y. Yu, Z. Yang, K. Vu, T. Nguyen, A. Mitchell, B. LutherDavies, and S. Madden, "High Q factor chalcogenide ring resonators for cavity-enhanced MIR spectroscopic sensing," Opt. Express 23, 1996919979 (2015)

10. A. Joërg and J. Lumeau, "Fabrication of binary volumetric diffractive optical elements in photosensitive chalcogenide AMTIR-1 layers," Opt. Lett. 40, 3233-3236 (2015).

11. J. Lumeau and L. B. Glebov, "Effect of the refractive index change kinetics of photosensitive materials on the diffraction efficiency of reflecting Bragg gratings", Applied Optics 52(17), 3993-3997 (2013).

12. C. Kress and P. Meyrueis, Applied Digital Optics: From Micro-optics to Nanophotonics, Wiley, 2009.

13. O. Ripoll, V.Kettunen and H. Herzig, "Review of iterative Fouriertransform algorithms for beam shaping applications," Optical Engineering 43, 2549-2556 (2004).

14. N. Yoshikawa and T. Yatagai, "Phase optimization of kinoform by simulated annealing", Applied optics 33, 863-868 (1994). 